\title{
The Research on Current Situation and Promotion Strategy of Health Qigong in Colleges and Universities
}

\author{
Ping Liu ${ }^{\mathrm{a}}$, Xiangdong Liu \\ Pingxiang University, Jiangxi 337055, China \\ a55057169@qq.com, b342099147@qq.com
}

\begin{abstract}
Health Qigong is one of the outstanding heritage of our national traditional culture, it has been promoted for more than 10 years, in colleges and universities have also been constantly developing and growing. However, at present, there are some shortcomings in the popularization and popularization of fitness qigong in colleges and universities. It is necessary to carry out in-depth study on these problems, and then put forward the countermeasures to promote the development of the project to play the role of fitness qigong in building and strengthening college students' physical.
\end{abstract}

Keywords: fitness qigong, university, promotion, strategy.

\section{Introduction}

Health Qigong is an excellent legacy in the traditional culture of our country. It combines the theories of philosophy, medicine and culture to play a significant role in promoting people's health care and so on. At present, fitness Qigong has been in the world to gradually promote and develop, but also by the people of the world sought after and love. As one of the highest forward positions in imparting the essence of human culture, colleges and universities also carry the mission of inheriting and carrying forward the outstanding national cultural heritage of China, revealing the scientific nature of fitness qigong; on the other hand, it is also responsible for cultivating and improving college students' attention to sports consciousness and habit Obligations and responsibilities in the new situation, the higher education in colleges and universities put forward higher requirements, in addition to promoting the physical health of college students, but also to develop students consciously exercise habits and the formation of lifelong sports awareness. In the choice of teaching content also requires focus on the combination of competitive and national character, promote our national traditional sports. Therefore, the fitness qigong as a strong national culture with the characteristics of the traditional national sports, Its fitness and the role of fitness to meet the needs of college students sports. China began to promote health Qigong in the country since 2003, many colleges and universities have also opened such courses, but the current college exercise Qigong curriculum is not standardized, there are many problems, for this phenomenon is necessary for college health Qigong To carry out the status quo to conduct a certain investigation and research, and put forward the promotion of strategies and recommendations to achieve fitness Qigong in college students on the physical health and the promotion of sports awareness.

\section{The research objects and methods}

\subsection{The research objects}

The research object of this paper is the status quo and problems of health qigong in 8 colleges and universities in Jiangxi province.

\subsection{The research methods}

(1) Literature method

Through the journals online access and download the national traditional sports and fitness qigong relevant papers and writings, a comprehensive understanding of the research situation of fitness qigong, drawing on previous research methods and research results for the study to provide a theoretical basis. 
(2) Questionnaire survey method

A total of 400 questionnaires were collected from 8 university students in Jiangxi Province. There were 50 schools and 486 questionnaires. Among them, 370 valid questionnaires were valid and the effective recovery rate was $95.8 \%$. Mainly for students to learn the work of fitness Qigong and health Qigong view of the investigation

(3) Expert Interview

In the questionnaire survey, the eight colleges and universities in charge of physical education department leaders and as a fitness qigong course full-time teachers in face-to-face interviews, for the current colleges and universities to carry out fitness qigong problems and deficiencies widely solicited opinions.

\section{The research results and analysis}

\subsection{Colleges and universities to carry out the form of fitness qigong teaching}

At present, with the continuous expansion of colleges and universities and the increase in the number of schools in the form of fitness Qigong to open the form of public sports elective courses, sports associations and sports clubs. Through the survey of eight colleges and universities in Jiangxi Province, it was found that seven colleges and universities had set up fitness qigong elective courses with a proportion of $87.5 \%$. All schools had set up corresponding martial arts associations or fitness qigong associations. There were also two colleges Built a fitness qigong club, the main recruiting interested students after school training.

\subsection{Students' awareness of fitness qigong}

As we all know, interest is the best teacher, interest is the most direct motivation for college students to learn Qigong. In the survey of 400 college students for fitness Qigong survey: 15.1\% of students are interested; $40.5 \%$ of students are interested; only $23 \%$ of students are not interested in fitness qigong. It can be seen that after 10 years of development, the popularity of college students in Jiangxi Province is very high. Second, $11 \%$ of the 400 people surveyed had come into contact with fitness qigong or were practicing the project.

\subsection{The development of fitness qigong course in colleges and universities}

The teaching of fitness qigong course in colleges and universities is mainly through the form of sports elective courses to provide services to freshman and sophomore students. Some school courses are called health qigong. The two major semesters are the main course of the course. The main contents of the examination are the four sets of exercises, such as Ba Duan Jin, Wu Qin Xi, Yi Jin Jing and Guiding Health.

\subsection{PE teachers on the degree of fitness Qigong degree of understanding}

In an interview with 15 sports teachers in eight colleges and universities, $86.7 \%$ of the teachers thought that Qigong had a great impact on the construction and development of the school itself, and only $13.3 \%$ thought that there was a certain impact. All teachers believe that fitness qigong to enhance the physical fitness of college students have a very good role in promoting.

\subsection{Lack of fitness qigong in colleges and universities}

In the form of examination mainly in the mastery of student technology scoring, there is no relevant theoretical examination, usually teaching the lack of traditional Chinese sports culture, resulting in many students just a simple learning action, and not from the heart to master and understand the health Qigong Function and efficacy. In the interview with 15 teachers, 66.7\% of the teachers believe that in the future teaching and examination methods need to be some reform. In addition, $80 \%$ of the teachers reflect the school facilities to carry out fitness qigong facilities is not enough, many are and aerobics, martial arts and other projects common venues, there is no special practice venues, but also $54 \%$ of students also think that school sports facilities Lack of enough to meet the needs of students physical exercise. Through the survey found that health Qigong professional teachers are scarce, and the Provincial Sports Bureau to carry out the relevant training is also less, resulting in a lot of college students Qigong from martial arts, teachers are relatively weak. 


\section{Conclusions and recommendations}

\subsection{Conclusion}

(1) At present, colleges and universities to carry out fitness qigong teaching in the form of public sports elective courses, fitness qigong group associations, sports clubs.

(2) More than half of the students are interested in fitness qigong, and some of them are already in contact with or are practicing the project.

(3) Development of Health Qigong Course in Colleges and Universities.

The teaching of the course of fitness qigong in colleges and universities is mainly through the two semesters of sports elective courses to provide services to freshman and sophomore students. The main contents of the examination include Ba Duan Jin, Wu Qin Xi, Yi Jin Jing and Guiding Health And so on four sets of exercises, focusing on students to master the skills of the situation.

(4) Physical education teachers on the fitness qigong course of knowledge is high, the general task of fitness Qigong to enhance the physical fitness of college students have a very good role in promoting.

(5) Colleges and universities to carry out fitness Qigong courses exist examination methods, the lack of relevant theoretical teaching, specialized fitness Qigong site facilities and full-time teachers and other weak.

\subsection{Strategies and Suggestions on the Promotion of Health Qigong in Colleges and Universities}

(1) Fitness Qigong in order to get a smooth and extensive development of colleges and universities, first of all to the university leaders and teachers and students of the recognition, to let the school leadership to guide the work of fitness Qigong students, know how to set up a new curriculum is the reform of college physical education Inevitable trend, but also an important indicator of the quality of teaching evaluation.

(2) Colleges and universities can set up public sports elective courses, to improve the fitness Qigong teaching materials, a clear outline of teaching, teaching objectives, the way the examination of a certain degree of reform and innovation.

(3) Attach importance to the role of colleges and universities, associations and clubs in the promotion of fitness Qigong, to contribute to the spread of fitness qigong.

(4) To strengthen the construction of fitness Qigong teachers, the introduction of professionals, pay attention to the exchange of teachers training, increase the work of fitness Qigong declaration and research efforts;

(5) To guide colleges and universities to actively participate in and organize health qigong contest activities, increase the theoretical and technical exchanges between universities, and expand the influence of fitness qigong in colleges and universities to promote.

(6) Health Qigong as a good national traditional culture, in the promotion process should fully tap the health and qigong cultural and educational functions, and cultivate college students inherited the concept of traditional national culture, to promote national spirit, enhance national cohesion to contribute.

\section{References}

[1] Zhang Yunya. Health Qigong to promote the popularization of the status quo [J]. Journal of Shandong Institute of Physical Education. 2008 (24)

[2] Shan Hai Long. Fitness Qigong in Chengdu colleges and universities to carry out the status quo [D]. Chengdu: Sichuan Normal University. 2014

[3] Xiang Hanping. Fitness Qigong in the development of ordinary colleges and universities and development countermeasures [J]. Journal of Wuhan Institute of Physical Education. 2013 (47) 